\title{
Scanning coherent diffractive imaging methods for actinic EUV mask metrology
}

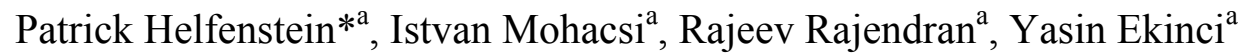 \\ Paul Scherrer Institute, 5232 Villigen PSI, Switzerland
}

\begin{abstract}
For the successful implementation of extreme ultraviolet (EUV) lithography in the upcoming technology nodes, a major challenge to overcome is the stable and reliable detection and characterization of mask defects. We have recently presented a reflective mode EUV mask scanning lensless imaging tool (RESCAN) which is installed at the XIL-II beamline of the Swiss Light Source and showed reconstructed aerial images of test patterns on EUV masks. RESCAN uses scanning coherent diffractive imaging (SCDI) methods to obtain actinic aerial images of EUV photomasks and was designed for $20 \mathrm{~nm}$ on-wafer resolution. Our SCDI algorithm reconstructs the measured sample by iteratively solving the phase-problem using over-determined diffraction data gathered by scanning across the specimen with a finite illumination. It provides phase and amplitude aerial images of EUV photomasks with high resolution without the need to use high NA (numerical aperture) lenses. Contrary to scanning microscopy and full-field microscopy, where the resolution is limited by the spot size or NA of the lens, the achievable resolution with our method depends on the detector noise and NA of the detector. To increase the resolution of our tool, we upgraded RESCAN with a new detector and algorithms. Here we present the results obtained with the new tool that is capable of up to $10 \mathrm{~nm}$ on-wafer resolution. We believe that the realization of our prototype marks a significant step towards overcoming the limitations imposed by methods relying on imaging optics and shows a viable solution for actinic mask metrology.
\end{abstract}

Keywords: mask metrology, actinic inspection, EUV, CDI, lensless imaging

\section{INTRODUCTION}

Extreme ultraviolet lithography (EUVL) has been developed over the past decades and is considered the most likely candidate for upcoming technology nodes with the industry getting ready for high-volume manufacturing. ${ }^{1}$ However, the key issue to solve, apart from the source power performance gap, still remains the reliable detection and repair of mask defects. ${ }^{2,3}$ Actinic mask metrology, being regarded as an essential part of the EUV mask infrastructure, is a major challenge and methods beyond the state-of-the-art are needed. The established metrology tools such as scanning electron microscopy have the disadvantage of being non-actinic, i. e. their imaging response from the absorber, multilayer, and pellicle varies from that of the scanner due to the use of electrons and photons at wavelengths different from the EUV design wavelength. ${ }^{4}$ Phase defects (resulting from bumps and pits in the substrate below the multilayer) are particularly problematic as these are elusive to detect and difficult to repair. ${ }^{5}$

Here we report on the results of our EUV mask scanning lensless imaging tool (RESCAN) which uses a scanning coherent diffraction imaging (SCDI) method. ${ }^{6}$ SCDI methods such as ptychography have been invented a long time ago but where long considered impractical for experimental use due to the stringent restrictions of early algorithms. Fortunately, recent advances in the phase retrieval algorithms ${ }^{7-10}$ have made SCDI a simple, versatile, and effective technique in applications ranging from hard X-rays to visible light and electrons. It is also used for EUV mask imaging by many groups ${ }^{11-13}$ and to overcome the resolution limitations of lens-based actinic microscopes. ${ }^{14}$ SCDI uses scattering data recorded with a pixel detector obtained through scanning a finite illumination (probe) across the sample (object) to be imaged. Each subsequent position is chosen so that it overlaps with the previous position to a certain extent. This redundancy in the data is used to retrieve the missing phase and thereby enables robust reconstruction of the complex aerial image of the sample without the need for a reference beam or structure. It enables to simultaneously obtain both phase and amplitude information of the sample with a 2D scan, whereas lens-based imaging methods need through focus scans in order to extract the phase information. Moreover, it has a large depth-of-focus, since the spot size is relatively insensitive to small changes in the large focal distance of the condenser. Since it is a lensless method, it does not need

Extreme Ultraviolet (EUV) Lithography VII, edited by Eric M. Panning, Kenneth A. Goldberg. Proc. of SPIE Vol. 9776, 97761F · @ 2016 SPIE · CCC code: 0277-786X/16/\$18 · doi: 10.1117/12.2219937 
expensive or difficult optics. The resolution of SCDI is typically limited by the NA of the detector, which defines the maximum spatial frequency information acquired. We note that the resolution can be also limited by various other parameters such as the source stability, illumination parameters, scanning method, mechanical instabilities, detector noise, detector pixel number, etc.

Since SCDI offers the aforementioned advantages, it can be a powerful method for actinic EUV mask metrology. We developed special algorithms for EUV mask imaging, which evolved from classical SCDI methods towards the specific needs of EUV mask imaging by addressing specific difficulties of EUV mask metrology and making use of specific advantageous aspects of it. In this paper we describe our tool and show its imaging performance.

\section{EXPERIMENTAL SETUP}

The experimental setup of RESCAN is installed at the XIL-II beamline of the Swiss Light Source (SLS). The XIL-II beamline was designed for interference lithography and provides a spatially coherent beam of EUV light $(\lambda=13.5 \mathrm{~nm})$ with a bandwidth of $4 \%$. The RESCAN chamber is connected to the interference lithography chamber, sharing the same optical table. It incorporates all the necessary components needed for SCDI from EUV masks, i.e. EUV beam manipulation optics (focusing, monochromatization) for probe generation, a high resolution sample handling stage and a pixel detector. Since the XIL-II beamline is optimized for interference lithography applications the conditions for lensless metrology are far from ideal. The large bandwidth, in particular, severely limits the experiments.

In Figure 1 (a) the schematics of the experimental chamber are shown. The beam enters the chamber from the left and is then focused by a Fresnel zone plate (FZP) with a focal length of $142 \mathrm{~mm}$. To ensure that the focus coincides with the pinhole plane after being reflected from the Si/Mo multi-layer (ML) mirror, the FZP was mounted on a piezoelectric $x-y-z$ stage and the position adjusted accordingly. In the current setup, no order sorting aperture (OSA) was used and the focused beam is aligned to the $\sim 4 \mu \mathrm{m}$ pinhole (cf.

Figure 1 (b)) by positioning the ML mirror using two piezoelectric motors. Using a small pinhole placed before allowing the incident illumination to be diffracted on the sample ensures that only the first order diffraction of the FZP reaches the sample. Due to the wavelength dependent focusing properties of the FZP, the bandwidth is thus reduced from the initial $4 \%$ to $\sim 0.5 \%$. The pinhole and aperture were manufactured on a silicon nitride membrane using electron beam lithography, metal deposition and partial etching of the nitride support. Both the FZP and the pinhole assembly were manufactured in-house. RESCAN was designed as a versatile tool for scattering lensless imaging techniques at EUV wavelengths and further results have been published. ${ }^{15}$

After passing through the pinhole, the monochromatized beam expands and illuminates the sample at an angle of $6^{\circ}$ with a spot size of about $8 \mu \mathrm{m}$. The pinhole-to-sample distance is $\sim 0.7 \mu \mathrm{m}$. The reflected beam from the sample passes through a $300 \times 300 \mu \mathrm{m}^{2}$ aperture on the pinhole assembly (Figure 1b) and reaches the detector which is set at a distance of $\sim 77 \mathrm{~mm}$ from the sample. The diffraction patterns are captured by a $2048 \times 2048$ pixel CCD camera (Princeton Instruments, PI-MTE2048B) with the exposure time for the data presented here set to $250 \mathrm{~ms}$, which leads to the best use of the 16 bit dynamic range of the CCD detector without saturation. The sample is mounted on a piezoelectric $x-y-z$ stage operated in closed-loop configuration with an accuracy of a few nanometers. The diffraction patterns are collected in sequence of moving the sample, actuating the shutter (triggered by the CCD controller) and reading out the CCD data in an automatic manner using EPICS software. The throughput bottleneck is the frame rate of the detector which is below $1 \mathrm{~Hz}$.

The test sample used for the data presented here (cf.

Figure 2) was fabricated in house by spin-coating HSQ resist with a thickness of $\sim 60 \mathrm{~nm}$ onto a Si wafer coated with a Si/Mo multilayer. The HSQ absorber structures were then patterned by electron beam lithography and consist of several gratings with programmed defects, a Siemens star, L-shapes, square patterns and several more complicated structures. The four gratings shown have a half-pitch (hp) of $1 \mu \mathrm{m}, 500 \mathrm{~nm}, 250 \mathrm{~nm}, 100 \mathrm{~nm}$ from bottom to the top and left to right, respectively. We note the sample is not a state-of-the-art EUV mask and exhibits poor contrast due to the high EUV-transparency of the HSQ resist. 

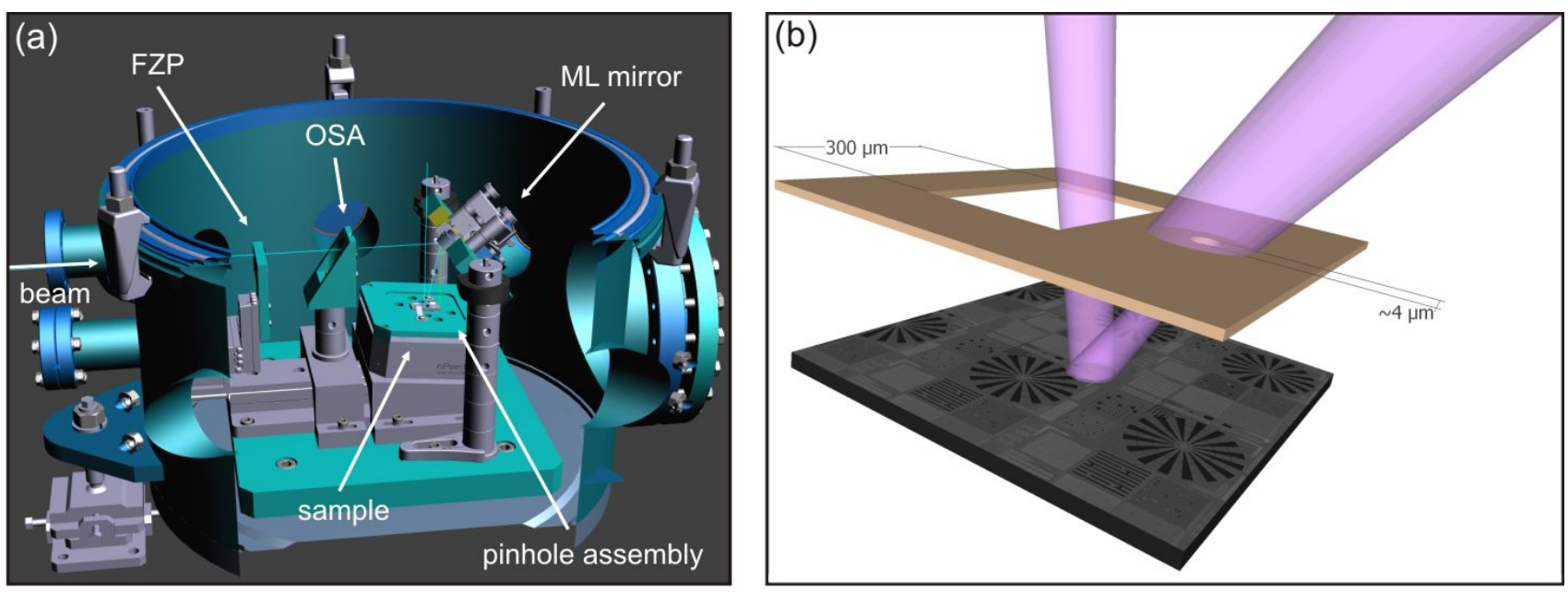

Figure 1 Schematic view of the experimental setup. (a) cross-section of the RESCAN chamber showing the beam path (light blue line). After entering, the beam is focused by a Fresnel zone plate (FZP) and the reflected from a multi-layer (ML) mirror through a pinhole onto the sample. The resulting diffraction patterns are recorded on a CCD detector which is not shown in this figure. The order sorting aperture (OSA) was not used for the present experiment. (b) Close-up view of the pinhole assembly. The focused beam with $4 \%$ bandwidth passes through a $\sim 4 \mu \mathrm{m}$ pinhole whereby the bandwidth is reduced to $0.5 \%$.

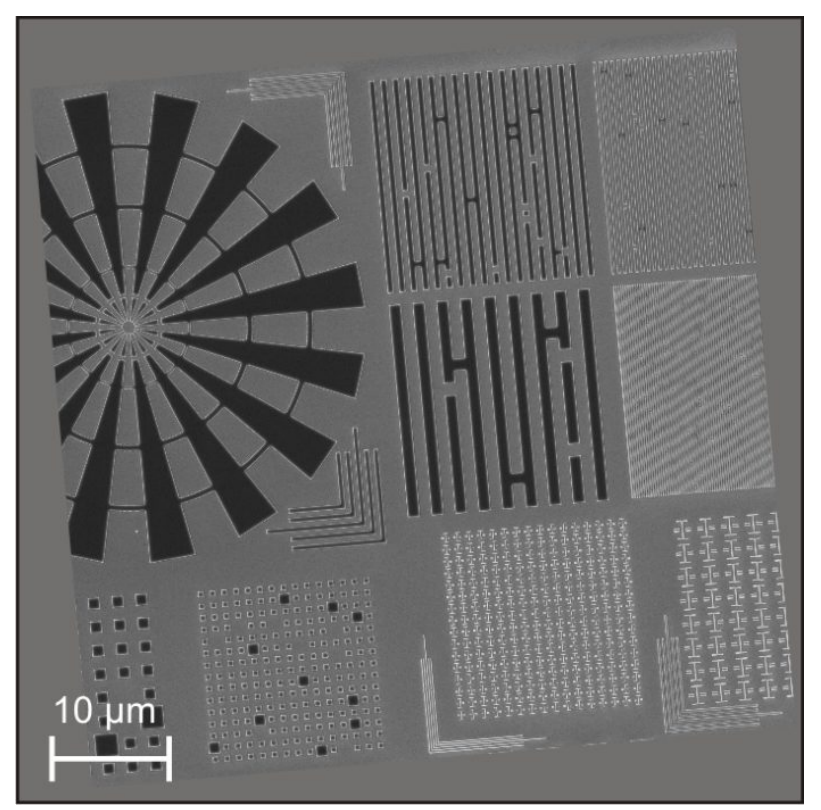

Figure 2. SEM micrograph of the test sample. The HSQ absorber structures were spin-coated on a multilayer wafer and then patterned using electron beam lithography.

\section{RESULTS}

Figure 3 shows both the magnitude and phase of the reconstructed aerial image from our test sample shown in 
Figure 2. For this specific experiment, we acquired 220 diffraction patterns with a step size of $3 \mu \mathrm{m}$ between subsequent scan positions resulting in a $50 \times 50 \mu \mathrm{m}^{2}$ field-of-view, thereby guaranteeing sufficient overlap for the used probe with a diameter of $\sim 8 \mu \mathrm{m}$. The positions were chosen with small randomness in $x$ and $y$ axes to ensure that they would not form a regular grid. If all scan positions lie on a perfectly regular grid, an additional degree of freedom is introduced into the reconstruction process which allows any function with the same periodicity as the scanned grid as valid solution for reconstruction and leads to artifacts. ${ }^{9}$

For this reconstructed aerial image, we used a SCDI method based on the difference map algorithm (DMA). ${ }^{8}$ In this approach, both the incident illumination on the sample (probe) and the sample structure (object) are iteratively reconstructed at the same time. The phase information is retrieved by alternately updating the probe and the object and searching for a consistent solution within the following two constraint sets:

i) Overlap constraint: The data in the overlapping regions has to agree

ii) Fourier constraint: The reconstructed intensity must be equal to the measured intensity

Assuming that the measured intensity signal can be decomposed into probe and object, the algorithm converges to a valid solution. In the presence of noise (i.e. read out noise of the detector and photon shot noise) and under other experimental imperfections (position inaccuracy, limited monochromaticity, etc.) any local minimum (i. e. any solution that marks a point in the intersection of the two constraint sets) is a valid solution. Therefore, the algorithm never fully converges, but alternates between possible solutions near the minimum, leading to some artifacts in the reconstructed aerial image. To mitigate this problem, the reconstructed aerial image is averaged over a number of iterations. In the present case, we obtained the final aerial image by running 300 iterations in total and averaging from 240-300 using every $4^{\text {th }}$ iteration. Before reconstruction, a pre-recorded background (dark signal) was subtracted from each dataset and the resulting image cropped in order to center the diffraction pattern. As we noticed that the CCD detector is misaligned with respect to the sample $x$ and $y$ axes, we rotated the coordinate system offline to accommodate for this offset.

From the resulting reconstructed aerial image we calculated the achieved resolution by taking various $10 \%-90 \%$ edge profiles from the gratings as well as the Siemens star and arrived at an average value over the full field of view of $60 \mathrm{~nm}$ (corresponding to $15 \mathrm{~nm}$ on wafer). Under the present experimental configuration (the NA of the detector and the $.5 \%$ beam monochromaticity, etc.) this is a remarkable achievement as the NA limited resolution is $\sim 51 \mathrm{~nm}$ (on mask) and we obtained a value close to the theoretical limit.

In addition, we employed a super-resolution method in order to increase the resolution beyond the limit set by the detector. We created a virtual detector with larger NA (i.e. more pixels) by zero-padding each diffraction pattern. ${ }^{16}$ During the reconstruction, the pixels in the zero-padded area were allowed to take any value, thereby enabling the algorithm to extrapolate the measured diffraction data into this extended region. We then measured the 10\%-90\% edge response for the smallest grating with $100 \mathrm{~nm} \mathrm{hp} \mathrm{(cf.} \mathrm{the} \mathrm{inset} \mathrm{in}$ Figure 3 (a)). By extending the original diffraction patterns from $1500 \times 1500$ pixels to $3000 \times 3000$ pixels, the pixel size of the reconstructed aerial image was reduced from $\sim 51 \mathrm{~nm}$ to $\sim 26 \mathrm{~nm}$. As can be seen in

Figure 4, the resolution calculated from the edge response averaged over 5 lines could be increased to $40 \mathrm{~nm}$ (corresponding to $10 \mathrm{~nm}$ on wafer) in this specific area. 

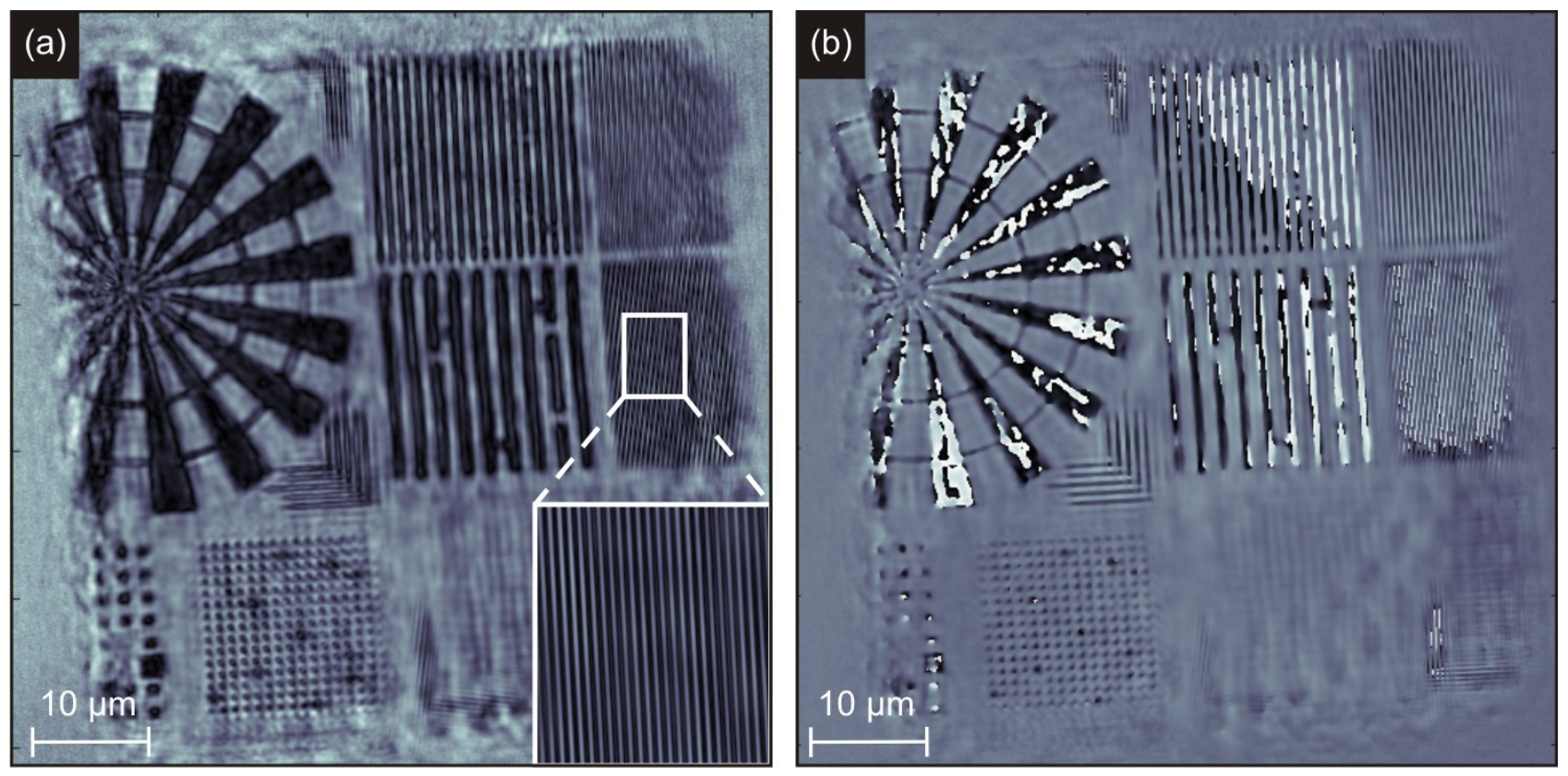

Figure 3 Reconstructed aerial image of a sample (a) Magnitude map of the image. The inlay shows a close up of the smallest grating with $100 \mathrm{~nm} \mathrm{hp}$. (b) Phase map of the image. The jumps in value stem from the fact that the phase can only be reconstructed modulo $2 \pi$ and is therefore wrapped around $-\pi$ to $\pi$.
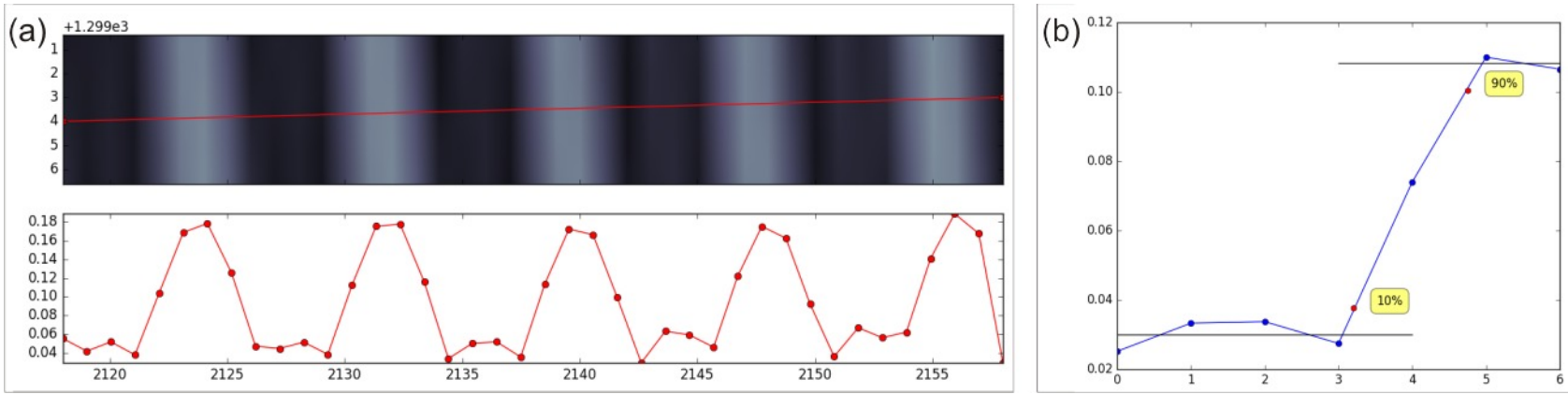

Figure $410 \%-90 \%$ edge response of the $100 \mathrm{~nm}$ hp grating (a) close-up view of 5 lines from the grating shown in the inset of Figure 3 (a) (top) and the corresponding cross section (bottom) (b) 10\%-90\% edge response averaged over the 5 lines where the horizontal lines at the bottom and top mark the $0 \%$ and $100 \%$ values, respectively.

\section{CONCLUSIONS AND OUTLOOK}

Using RESCAN, we have been able to show an average on-wafer resolution of $15 \mathrm{~nm}$ over a $50 \times 50 \mu \mathrm{m}^{2}$ field of view. Moreover, using zero-padding of the accumulated diffraction patterns and allowing the difference map algorithm to extrapolate the measured data, we have been able to achieve an on-wafer resolution of $10 \mathrm{~nm}$ which is beyond the NA resolution limit of the current setup that is limited by the distance of the detector to the mask. In the future, we would like to increase the resolution by increasing the physical NA. 
The reconstructed aerial images still feature some artifacts. We attribute this to the experimental parameters, such as monochromaticity, in combination with the algorithms. In the near future, a new chamber will be installed, providing better beam conditions and experimental control to mitigate these effects. A major improvement will be avoiding the use of FZP and pinhole and focusing the beam with a simple reflective condenser enabling more flexibility and better control of the experimental parameters. Moreover, we will further improve the algorithms in order to minimize the artifacts.

In addition, a metrology branch at the XIL-II beamline of SLS is under construction. It will be a dedicated tool for further development of the lensless microscopy methods for actinic EUV mask metrology. Future work includes studying the effects of the experimental parameters on the performance of EUV imaging. Further hardware development, such as fast frame rate detectors, will significantly increase the throughput.

\section{ACKNOWLEDGEMENTS}

We would like to express our gratitude to all the members of our group at PSI, the XIL-II beamline and also Manuel Guizar-Sicairos for technical assistance and fruitful discussions. Furthermore, we thank the Swiss National Science Foundation (SNF) and NuFlare Technology Inc., Japan, for financial support.

\section{REFERENCES}

[1] Hendrickx, E., Gronheid, R., Hermans, et al., "Readiness of EUV Lithography for Insertion into Manufacturing: The IMEC EUV Program", Journal of Photopolymer Science and Technology 26, 587-593 (2013).

[2] Uzzel, D., Garetto, A., Magnusson, K., Tabbone, G., "A novel method for utilizing AIMS"M to evaluate mask repair and quantify over-repair or under-repair condition", Proc. SPIE 8880, 888029 (2013).

[3] Goldberg, K. A., Mochi, I., Benk, M., et al., "Commissioning an EUV mask microscope for lithography generations reaching $8 \mathrm{~nm}$ ”, Proc. SPIE 8679, 867919 (2013).

[4] Mochi, I., Goldberg, K. A., La Fontaine, et al., "Actinic imaging of native and programmed defects on a full-field mask", Proc. SPIE 7636, 76361A (2010).

[5] Goldberg, K., Mochi, I., "Wavelength-Specific Reflections: A Decade of EUV Mask Inspection Research", International Workshop on EUV Lithography (2009).

[6] Hoppe, W., "Beugung im inhomogenen Primärstrahlwellenfeld. I. Prinzip einer Phasenmessung von Elektronenbeungungsinterferenzen". Acta Crystallographica A 25 (4), 495 (1969).

[7] Rodenburg, J. M. and Faulkner, H. M. L., "A phase retrieval algorithm for shifting illumination", Applied Physics Letters 85(20), 4795 (2004).

[8] Thibault, P., Dierolf, M., Menzel, A., Bunk, O., David, C., and Pfeiffer, F., "High-resolution scanning X-ray diffraction microscopy", Science (New York, N.Y.) 321, 379-82 (2008).

[9] Thibault, P., Dierolf, M., Bunk, O., et al., "Probe retrieval in ptychographic coherent diffractive imaging", Ultramicroscopy 109, 338 (2009)

[10] Maiden, A. M. and Rodenburg, J. M., "An improved ptychographical phase retrieval algorithm for diffractive imaging", Ultramicroscopy 109, 1256 (2009).

[11] Seaberg, M. D., Adams, D. E., Townsend, E. L., et al., "Ultrahigh $22 \mathrm{~nm}$ resolution coherent diffractive imaging using a desktop $13 \mathrm{~nm}$ high harmonic source", Opt. Express 19, 22470-9 (2011).

[12] Gardner, D. F., Zhang, B., Seaberg, M. D., et al., "High numerical aperture reflection mode coherent diffraction microscopy using off-axis apertured illumination", Opt. Express 20, 19050-9 (2012).

[13] Juschkin, L., Loetgering, L., Rudolf, D., et al., "Tabletop coherent diffraction imaging with a discharge plasma EUV source", Proc. SPIE 8849, 88490Y (2013)

[14] Wojdyla, A. J., Benk, M. P., Goldberg, K. A., "Fourier Ptychography imaging for the study of EUV lithography photomasks", Proc. SPIE 9776 (2016)

[15] I. Mohacsi, P. Helfenstein, Rajeev Rajendran, and Y. Ekinci, "Scanning Scattering Contrast Microscopy for actinic EUV mask inspection", Proc. SPIE 9778 (2016)

[16] Maiden, A. M., Humphry, M. J., Zhang, F., and Rodenburg, J. M., "Superresolution imaging via ptychography", J. Opt. Soc. Am. A 28, 604 (2011) 